\title{
Influence of Light Intensity exercise During Pregnancy on Fetal Echocardiographic and Maternal Cardiovascular Measures
}

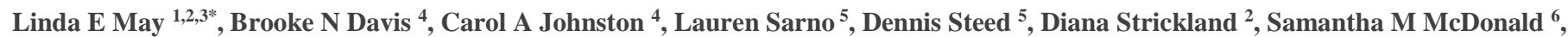
Breanne L. Wisseman ${ }^{3}$, Ed Newton ${ }^{2}$, Christy Isler ${ }^{2}$, Seonae Yeo ${ }^{7}$, Devon Kuehn ${ }^{2}$

${ }^{1}$ Department of Foundational Science and Research, ECU, Greenville, NC, USA

${ }^{2}$ Department of Obstetrics and Gynecology, ECU, Greenville, NC, USA

${ }^{3}$ Department of Kinesiology, ECU, Greenville, NC, USA

${ }^{4}$ Department of Human Development and Family Sciences, Greenville, NC, USA

${ }^{5}$ Department of Pediatrics, ECU, Greenville, NC, USA

${ }^{6}$ School of Kinesiology and Recreation, Illinois State University, Normal, IL, USA

${ }^{7}$ School of Nursing, University of North Carolina at Chapel Hill, NC, USA

*Corresponding Author: Linda E May, MS, PhD, FAHA, FACSM 1851 Mac Gregor Downs Rd Greenville, NC 27834.

Received Date: December 06, 2021; Accepted Date: December 13, 2021; Published Date: January 10, 2022

Citation: Linda E May, Brooke N Davis, Carol A Johnston, Lauren Sarno, Dennis Steed. et all (2022). Influence of Light Intensity exercise During Pregnancy on Fetal Echocardiographic and Maternal Cardiovascular Measures. J. Clinical Cardiology and Cardiovascular Interventions, 5(2); DOI:10.31579/2641-0419/237

Copyright: () 2022 Linda E May, This is an open-access article distributed under the terms of the Creative Commons Attribution License, which permits unrestricted use, distribution, and reproduction in any medium, provided the original author and source are credited.

\section{Abstract}

Background: Although moderate intensity maternal exercise is recommended during pregnancy, most women do not achieve this level.

Objective: This study aimed to determine the effects of supervised maternal light-intensity exercise on fetal cardiovascular measures in the $3^{\text {rd }}$ trimester.

Materials and Methods: We compared women participating in light-intensity (LI) stretching/breathing or no exercise $(\mathrm{CON})$ across $24+$ weeks of pregnancy Women with singleton pregnancies (<16 weeks), between 18-40 years, 18.5-34.99 $\mathrm{kg} / \mathrm{m}^{2} \mathrm{BMI}$, and having no chronic health conditions were eligible. LI did 150 minutes light-intensity weekly exercise; CON did normal daily activities, but no exercise. Maternal measures included resting heart rate (HR) and blood pressure (BP). Fetal measures included estimated fetal weight (EFW), ponderal index (PI), HR, along with right and left ventricular measures (e.g., stroke volume, cardiac output, ejection fraction) at 36 weeks' gestation. T-tests compared differences between groups; partial spearman rank correlations and regressions were performed to find relationships and predict fetal echocardiographic outcomes, controlling for fetal activity state.

Results: Of 50 pregnant women ( $n=26$ in LI, $n=24$ in CON), we found maternal exercise intensity influences fetal left ventricular heart flow, while maternal exercise duration influences fetal right ventricular outcomes. There were no differences $(p>0.05)$ in fetal cardiac anatomical measures. Lastly, light intensity exercise and duration of exercise lower maternal blood pressure in late pregnancy.

Conclusions: Light-intensity exercise is associated with improved fetal cardiac measures. Women benefit from light intensity exercise during pregnancy. Cardiovascular benefits from exercise for mother and child are dose dependent.

Keywords: pregnancy; light intensity exercise; maternal; fetal; echocardiography 


\section{Introduction}

Although exercise at recommended levels of 150 minutes/week, moderate intensity during pregnancy demonstrates maternal and fetal benefits and decreased gestational conditions [1-7], more than half of pregnant women fail to meet the recommended levels of prenatal physical activity [8]. While research demonstrates maternal exercise has a dose response with fetal heart outcomes[9] and light intensity (LI) exercises is one way to decrease sedentary time, research shows this to be a preference for Hispanics, black, and obese pregnant women [10-12]. Light intensity exercises, such as walking, yoga, deep breathing with stretching, have supported benefits, like decreased incidence of pre-eclampsia [13, 14]. Furthermore, in the obese population, light intensity exercise decreases the occurrence of pre-eclampsia, whereas moderate-to-vigorous exercise has had mixed results[15]. Benefits of light intensity exercise are thought to be a result of improving parasympathetic control without stimulating the sympathetic nervous system $[10,14,16]$. In addition to improved maternal cardiac autonomic control, other benefits associated with light intensity exercise while pregnant include decreased perceived stress, increased maternal antioxidant levels, lower C-Reactive Protein levels, and improved pregnancy outcomes [17-20]. Light intensity walking is associated with improved glucose control in pregnant women with gestational diabetes[21]. Similar to moderate intensity exercise, light intensity prenatal yoga is associated with increased vaginal deliveries and pain tolerance while laboring [22]. Prenatal light intensity exercise typically has high adherence during pregnancy, especially in the obese population[15].

Little is known about potential effects of light intensity exercise during pregnancy on offspring outcomes; further, most research on birth outcomes is from moderate intensity maternal exercise, including one study that notes prenatal yoga is associated with attenuated low birth weight babies[22]. Interestingly, earlier research, using self-reported maternal exercise, has showed a dose response relationship between prenatal exercise and offspring heart outcomes [9, 23]. We are uncertain of the influence of light intensity exercise during pregnancy on fetal heart outcomes.

To address this gap in knowledge, the purpose of the current study was to find the effects of supervised prenatal light intensity exercise on maternal and fetal cardiovascular measures in the $3^{\text {rd }}$ trimester of pregnancy. We hypothesized that light intensity exercise during pregnancy would show improved maternal resting heart measures and greater fetal cardiac function (i.e., increases in stroke volume and index, cardiac output and index, ejection fraction), but not anatomical changes (i.e., pulmonary and aortic valve diameters) at 36 weeks of gestation compared to fetuses of non-exercising women.

\section{Materials and Methods}

This study employed a secondary analysis of a randomized controlled exercise intervention trial at East Carolina University from 2015 to 2018. The aim of the secondary analysis was to find if there were differences between the light intensity stretching/breathing group and the no exercise group regarding maternal and fetal heart outcomes. All study protocols were approved by the East Carolina Institutional Review Board, and each participant signed an informed consent form prior to enrollment.

Pregnant women were recruited from local obstetric clinics in eastern North Carolina using brochures and email announcements. Women were eligible for this study if they 1) were < 16 weeks pregnant, 2) had a lowrisk, singleton pregnancy, 3) were between the ages of 18 and 40 years, 4) had a pre-pregnancy BMI between 18.5 and $34.9 \mathrm{~kg} / \mathrm{m}^{2}$ and 5) had physician clearance to participate in exercise. Exclusion criteria for this study included 1) use of alcohol, tobacco, or recreational drugs, 2) taking medications known to affect fetal growth (e.g., antidepressants), 3) exhibited any contraindications for exercise during pregnancy, according to American College of Obstetricians and Gynecologists (ACOG) guidelines [24], or 4) were diagnosed with pre-existing diabetes mellitus (Type 1 or 2), hypertension, cardiovascular disease, or another comorbidity known to effect fetal growth and well-being (e.g. systemic lupus erythematous).

Randomization for both studies was performed by the principal investigator using a random sequence generator (GraphPad, San Diego, CA) and allocation was concealed prior to group assignment. One sonographer completed all ultrasounds and fetal echocardiograms and was blinded to participant group assignment; however, participants and exercise trainers were not blinded to group assignment due to the nature of the study.

All participants underwent a submaximal exercise treadmill test as validated for pregnant women by Mottola et al. [25]. During the treadmill test, oxygen consumption and carbon dioxide production were assessed via breath-by-breath analysis (Parvo Medics, TrueOne 2400, Sandy, UT) until volitional fatigue in order to assess $\operatorname{VO}_{2 \text { peak }}\left(\mathrm{ml} \mathrm{O}_{2} \times \mathrm{kg}^{-1} \times \mathrm{min}^{-1}\right)$. Maternal heart rate (HR) was measured continuously with a Polar FS2C HR monitor. This test was performed to calculate the target HR zones (THR). Target HRs for this study corresponded to maternal HRs obtained at $30-40 \% \mathrm{VO}_{2 \text { peak, }}$ reflecting light intensity [26].

Regular meetings differed by group. The light intensity exercise group completed a 50-minute exercise session 3 times per week for 24 weeks (16 weeks' gestation until delivery). Each session began with a 5-minute warm-up consisting of slow walking on a treadmill. Following this, the women were guided, via a trained staff member, through several breathing and stretching exercises. To maintain low intensity (30-40\% VO2peak), women were supervised by a trained staff member; maternal HR was monitored before, during, and after each training session using a Polar FS2C HR monitor (POLAR FS2c, Polar Electro Inc., Bethpage, NY). Exercise sessions were scheduled based on the participants' individual availability. However, the control group did normal daily activity, but no exercise. The daily activity and no exercise for the control group was verified by completion of the modifiable physical activity questionnaire (MPAQ) [27-30], validated for pregnant women, provided at least twice during the pregnancy (enrollment, 36 weeks' gestation). This questionnaire also provided participant demographic information (age, pre-pregnancy weight, number of pregnancies, number of live children, breastfeeding, highest degree obtained, race).[27, 28, 31] Results from the MPAQ after delivery was used to verify all participants' activity levels respective of their assigned group [27, 32].

In person exercise and/or MPAQ data was used to calculate average pregnancy exercise duration (minutes) and exercise volume $\left(\mathrm{MET} \cdot \mathrm{min} \cdot \mathrm{wk}^{-1}\right)$. Pregnancy MET $\cdot \mathrm{min} \cdot \mathrm{wk}^{-1}$ were quantified (frequency $X$ duration of session) then multiplied by the MET (metabolic equivalent) level of their assigned group, established by the Compendium for Physical Activity[33]. The total exercise duration (minutes) and volume (METmin) for all weeks were summed and averaged for the average pregnancy exercise duration ( $\mathrm{min} / \mathrm{wk}$ ) and volume $\left(\mathrm{MET} \cdot \mathrm{min}^{\circ} \mathrm{wk}^{-1}\right.$ ) values. Exercise session attendance was also tracked, via an electronic record and calculated by dividing the number of sessions attended by the total number of possible sessions in the participant's gestational period (16 weeks until delivery). Participants were considered "adherent" if their attendance was $\geq 80 \%$.

Our fetal echocardiographic outcomes were measured by one sonographer blinded to group allocation. At 34-36 weeks' gestation, an obstetrical ultrasound and fetal echocardiogram were performed between 12:00 and 1:00 pm at the university-affiliated outpatient clinic by a certified sonographer, blinded to group assignment, using a Logiq P5 ultrasound system (General Electric, Korea). These procedures are previously validated and found reliable in healthy, normal pregnancies 
producing accurate measurements of the fetal cardiac chamber dimensions and physiological measures of cardiac function.[34, 35] Obstetrical ultrasound and fetal echocardiogram were used to assess fetal anatomical and functional cardiac measures that included estimated fetal weight (EFW; grams), body length $(\mathrm{cm})$, pulmonary valve diameter, and aortic valve diameter. Body length $(\mathrm{cm})$ was calculated based on the standard formula 6.18+0.59Xfemur length (mm). [36] These outflow tract diameters were used to calculate outflow tract area $\left(0.785^{*}\right.$ diameter $\left.^{2}\right)$. The fetal echocardiogram was used to assess heart rate (beats $\cdot \min ^{-1}$ ), stroke volume $\left(\mathrm{mL} \cdot\right.$ beat $\left.{ }^{-1}\right)$, cardiac output $\left(\mathrm{L} \cdot \mathrm{min}^{-1}\right)$, pulmonary and aortic peak velocities $\left(\mathrm{cm} \cdot \mathrm{sec}^{-1}\right)$ and VTIs (velocity-time integral; $\mathrm{cm}$ ). Stroke volume (SV) was also calculated using VTI*outflow tract area since this has been shown to have improved prognostic value over ejection fraction.[37, 38] Cardiac output was calculated by multiplying stroke volume and heart rate. Stroke volume and cardiac output were additionally adjusted for body size via body surface area $\left(\mathrm{cm}^{3}\right)$ to calculate stroke index $\left(\mathrm{mL} \cdot\left(\mathrm{m}^{2}\right)^{-1}\right)$ and cardiac index $\left(\mathrm{L} \cdot\left(\mathrm{m}^{2}\right)^{-1}\right)$, respectively. Body surface area was calculated based on the Mostellar formula:[39] $\sqrt{((\text { fetal length } \cdot \text { fetal weight })} / 60)$. The ratio of right to left cardiac output and indices were calculated by dividing the right ventricular value by the left ventricular measures. The combined cardiac output and cardiac indices were calculated by summing the right and left ventricular measures. The fetal activity state (FAS), quiet or active, was determined by direct observation of the fetus' movement, heart rate, and heart rate pattern by the sonographer during the ultrasound recording [4042].

Maternal weight and height were assessed at 16 weeks of gestation. Prepregnancy weight was obtained from screening questions. Weight and height were measured in pounds and inches, respectively, and subsequently converted to their appropriate metric units and used to calculate body mass index using the standard formula $\left(\left(W t[k g] /\left(h t\left[m^{2}\right]\right)\right) \cdot[43]\right.$

Maternal demographic and pregnancy-related characteristics including gravida, parity, pre-pregnancy weight and height were abstracted from pre-screening eligibility questionnaires and verified with electronic health records; presence or absence of gestational diabetes mellitus (GDM), hypertensive disorders of pregnancy (e.g. pre-eclampsia, gestational hypertension), intrauterine growth restriction (IUGR), placental previa or abruption, delivery mode (i.e. vaginal, cesarean), gestational age (weeks), and Apgar scores at 1 and 5 minutes were recorded from electronic health records[44]. Pre-pregnancy body mass index (BMI) was calculated by dividing participant pre-pregnancy weight by the product of height squared $\left(\mathrm{kg} / \mathrm{m}^{2}\right)$. Preterm birth was defined as births $<37$ weeks' gestation.

Maternal physical measurements such as blood pressure (BP) and heart rate (HR) were measured at 16 and 36 weeks' gestation. Maternal resting blood pressure (BP) and heart rate (HR) were assessed in a sitting position. The BP was recorded using a manual sphygmomanometer at the brachial artery just proximal to the elbow (arm straight or in mild elbow flexion) with an appropriately sized cuff[45]. All measures were performed by trained research staff before each exercise session.

Between-group differences for maternal demographics, pregnancyrelated and maternal factors, and fetal cardiac outcomes were found using two-tailed independent t-tests if data were normally distributed or MannWhitney U tests for non-normally distributed data. Spearman's rank correlation tests were also used to assess correlations between maternal exercise, expressed as a continuous variable (MET·min·week ${ }^{-1}$ ) and fetal cardiac outcomes; this analysis was stratified by fetal activity state (FAS). Linear regression analysis was performed to assess predictors of fetal heart measures. All models used a stepwise process, in which variables were eliminated until the best model was achieved. In order to find predictors of fetal heart function, we used 3 models: Model 1 controlled for fitness level (Peak VO2), 36 wk mHR, exercise volume (METmin/wk), FAS, and gravida; Model 2 controlled for fitness level (Peak VO2), 36 wk mSBP, exercise volume (METmin/wk), FAS, and gravida. In order to assess which component of exercise volume (frequency, intensity, duration) is the strongest predictor of fetal heart outcomes, we used Models 3, 4, and 5. Model 3 controlled for fitness level (peak VO2), pre-pregnancy BMI, exercise frequency, exercise intensity, exercise duration, and FAS. Model 4 controlled for fitness level (peak VO2), maternal HR, exercise frequency, exercise intensity, exercise duration, FAS, and gravida. Model 5 controlled for fitness level (peak VO2), maternal HR, exercise frequency, exercise intensity, exercise duration, FAS, and gender. With alpha set at 0.05 and power of $80 \%$, sample size calculations determined we would need 30 total participants (15 per group) in order to detect differences between groups in the primary outcome of fetal heart rate. Excluding between group differences in the quiet state, all analyses had sufficient power to detect group differences.

\section{Results}

The analytical sample consisted of a convenience sample of 50 women, 26 in the light intensity exercise group and 24 in the control group that did not exercise. The average participant was 30 years old, overweight (BMI 26.1 \pm 5.78 ), unfit (resting $\mathrm{HR}=95 \mathrm{bpm}$ and $21.7 \mathrm{~mL} / \mathrm{min} \mathrm{VO} 2$ peak), and pregnant with her second child.

Maternal descriptors (e.g., pre-pregnancy BMI, 16- and 36-week blood pressure) were similar between groups (Table 1). There were no differences in fitness level between groups at enrollment based on resting HR and relative oxygen consumption (Table 1). However, a MannWhitney $U$ test revealed a significant between-group difference for maternal exercise intensity, duration, and volume (Table 1). There were no differences between groups regarding pregnancy outcomes (Table 1). Infant characteristics at birth were similar, except the light intensity group had babies about 400 grams lighter than non-exercising controls (Table 1). We found no significant differences in fetal heart measures between groups (Table 2).

\begin{tabular}{|l|l|l|l|}
\hline & Control & Light Intensity & p-value \\
\hline Maternal Characteristics & & & \\
\hline Pre-Pregnancy & & & \\
\hline Maternal Age (years) & $30.29(4.05)$ & $29.73(4.59)$ & 0.65 \\
\hline Pre-Pregnancy BMI & $27.32(6.68)$ & $24.93(4.66)$ & 0.15 \\
\hline Relative Peak $\mathrm{VO}_{2}(\mathrm{~mL} / \mathrm{kg} / \mathrm{min})$ & $21.12(3.92)$ & $22.22(4.07)$ & 0.34 \\
\hline *Gravida & $2.0(1,3)$ & $2.0(1,4)$ & 0.77 \\
\hline
\end{tabular}




\begin{tabular}{|c|c|c|c|}
\hline *Parity & $1.0(0,2)$ & $0.0(0,2)$ & 0.39 \\
\hline \multicolumn{4}{|l|}{16 weeks' Gestation } \\
\hline Resting HR (bpm) & $95.29(18.45)$ & $94.20(15.76)$ & 0.83 \\
\hline Resting Systolic Blood Pressure (mmHg) & $118.48(4.98)$ & $115.33(8.88)$ & 0.15 \\
\hline Resting Diastolic Blood Pressure (mmHg) & $77.90(6.65)$ & $73.33(11.26)$ & 0.10 \\
\hline \multicolumn{4}{|l|}{36 weeks' Gestation } \\
\hline Resting HR (bpm) & $96.77(18.62)$ & $94.32(15.06)$ & 0.62 \\
\hline Resting Systolic Blood Pressure (mmHg) & $117.55(8.23)$ & $115.60(10.77)$ & 0.49 \\
\hline Resting Diastolic Blood Pressure (mmHg) & $76.27(10.18)$ & $73.40(11.50)$ & 0.37 \\
\hline \multicolumn{4}{|l|}{ 2nd \& 3rd Trimester } \\
\hline Exercise Intensity (MET) & $2.29(0.61)$ & $2.71(0.23)$ & 0.004 \\
\hline Exercise Duration (min/wk) & $52.53(55.68)$ & $129.25(44.91)$ & $<0.001$ \\
\hline Exercise Volume (METmin/wk) & $140.23(160.58)$ & $327.37(122.56)$ & $<0.001$ \\
\hline \multicolumn{4}{|l|}{ Pregnancy Outcomes } \\
\hline \multicolumn{4}{|l|}{ Pregnancy Complications } \\
\hline GDM & $4.2 \%(1 / 24)$ & $15.4 \%(4 / 26)$ & 0.35 \\
\hline HDP & $0 \%(0 / 24)$ & $7.7 \%(2 / 26)$ & 0.49 \\
\hline IUGR & $0 \%(0 / 24$ & $3.8 \%(1 / 26)$ & 1.00 \\
\hline Placental previa or abruption & $4.2 \%(1 / 24)$ & $3.8 \%(1 / 26)$ & 1.00 \\
\hline Preterm Birth & $12.5 \%(3 / 24)$ & $11.5 \%(3 / 26)$ & 1.00 \\
\hline Delivery Mode & & & 0.75 \\
\hline Vaginal delivery & $75.0 \%(18 / 24)$ & $69.2 \%(18 / 26)$ & \\
\hline C-section & $25.0 \%(6 / 24)$ & $30.8 \%(8 / 26)$ & \\
\hline \multicolumn{4}{|l|}{ Infant Characteristics } \\
\hline Gestational Age & $38.98(1.33)$ & $39.04(1.61)$ & 0.89 \\
\hline Apgar at 1 minute & $7.48(2.13)$ & $8.20(1.26)$ & 0.17 \\
\hline Apgar at 5 minutes & $8.52(0.90)$ & $8.92(0.40)$ & 0.06 \\
\hline Fetal Weight $(\mathrm{kg})$ & $2.69(0.32)$ & $2.70(0.38)$ & 0.85 \\
\hline Fetal Length $(\mathrm{cm})$ & $45.82(1.61)$ & $46.61(2.83)$ & 0.25 \\
\hline Birth Weight (kg) & $3.50(0.55)$ & $3.10(0.44)$ & 0.03 \\
\hline \multicolumn{4}{|c|}{$\begin{array}{l}\text { Data presented as mean (SD) or percent (occurrences in group). *Due to non-normal distribution, we performed } \\
\text { Mann-Whitney U test for gravida and parity. BMI = body mass index, } \mathrm{VO}_{2}=\text { estimation of oxygen consumption, } \\
\mathrm{HR}=\text { heart rate, MET = metabolic equivalent for intensity of physical activities, METmin/wk = metabolic } \\
\text { equivalent in minutes per week, GDM = gestational diabetes mellitus, } \mathrm{HDP}=\text { hypertensive disorders of pregnancy, } \\
\text { IUGR = intrauterine growth restriction, C-section = cesarean operative delivery (either planned or unplanned). }\end{array}$} \\
\hline
\end{tabular}




\begin{tabular}{|c|c|c|c|}
\hline & Control & Light Intensity & p-value \\
\hline \multicolumn{4}{|l|}{ Fetal Heart Measures } \\
\hline Fetal HR (bpm) & $138.37(13.33)$ & $137.67(10.30)$ & 0.84 \\
\hline \multicolumn{4}{|l|}{ Right ventricular Heart Measures } \\
\hline RV Stroke Volume (L) & $1.03(0.18)$ & $1.04(0.17)$ & 0.80 \\
\hline RV Stroke Index (mL/kg) & $0.38(0.06)$ & $0.39(0.07)$ & 0.78 \\
\hline RV Cardiac Output (mL/min) & $141.40(16.38)$ & $144.31(20.61)$ & 0.61 \\
\hline RV Cardiac Index (mL/min/kg) & $52.71(8.17)$ & $52.53(9.11)$ & 0.95 \\
\hline RV Ejection Fraction (\%) & $60.52(16.14)$ & $56.14(15.17)$ & 0.34 \\
\hline \multicolumn{4}{|l|}{ Left ventricular Heart Measures } \\
\hline LV Stroke Volume (L) & $0.93(0.27)$ & $0.86(0.21)$ & 0.36 \\
\hline LV Stroke Index (mL/kg) & $0.34(0.08)$ & $0.33(0.06)$ & 0.43 \\
\hline LV Cardiac Output (mL/min) & $127.84(30.41)$ & $120.40(27.22)$ & 0.40 \\
\hline LV Cardiac Index (mL/min/kg) & $47.23(10.08)$ & $44.60(9.70)$ & 0.39 \\
\hline LV Ejection Fraction (\%) & $64.28(17.16)$ & $64.80(15.92)$ & 0.91 \\
\hline LV Fractional Shortening (\%) & $43.34(11.39)$ & $40.57(11.74)$ & 0.41 \\
\hline \multicolumn{4}{|l|}{ Valve Measures } \\
\hline Pulmonary Valve Area $\left(\mathrm{cm}^{2}\right)$ & $81.25(18.38)$ & $80.83(17.44)$ & 0.94 \\
\hline Pulmonary Valve Peak Velocity $(\mathrm{cm} / \mathrm{s})$ & $83.26(14.28)$ & $81.36(12.14)$ & 0.62 \\
\hline Pulmonary Valve Flow Velocity $(\mathrm{cm} / \mathrm{s})$ & $102.30(13.06)$ & $103.40(14.03)$ & 0.78 \\
\hline Aortic Valve Area $\left(\mathrm{cm}^{2}\right)$ & $53.22(10.73)$ & $50.39(14.18)$ & 0.44 \\
\hline Aortic Valve Peak Velocity $(\mathrm{cm} / \mathrm{s})$ & $102.26(17.38)$ & $96.68(10.59)$ & 0.19 \\
\hline Aortic Valve Flow Velocity $(\mathrm{cm} / \mathrm{s})$ & $113.11(28.21)$ & $107.72(17.51)$ & 0.44 \\
\hline Aortic Valve/Pulmonary Valve & $0.81(0.09)$ & $0.79(0.11)$ & 0.53 \\
\hline \multicolumn{4}{|l|}{ Right : Left Ventricular Associations } \\
\hline RV CO: LV CO (ratio) & $1.16(0.29)$ & $1.25(0.31)$ & 0.34 \\
\hline RV CI : LV CI (ratio) & $1.16(0.29)$ & $1.22(0.28)$ & 0.49 \\
\hline Combined CO (mL/min) & $269.25(37.27)$ & $264.71(36.94)$ & 0.69 \\
\hline Combined CI $(\mathrm{mL} / \mathrm{min} / \mathrm{kg})$ & $99.94(14.13)$ & $97.13(15.39)$ & 0.54 \\
\hline
\end{tabular}

Table 2: Fetal Echocardiographic measures between groups

We found a few notable correlations for maternal cardiovascular outcomes in late pregnancy related to exercise measures. Maternal exercise duration $(\mathrm{min} / \mathrm{wk})$ is $(\mathrm{p}=0.02)$ inversely but weakly $(\mathrm{rho}=-0.330)$ correlated to resting diastolic blood pressure at 36 weeks, and exercise duration has trends $(\mathrm{p}=0.06)$ of a weak negative association $(-0.272)$ with 36 wk SBP. Exercise Intensity (METs) was moderately (rho=0.419) correlated with 36 wk resting $\mathrm{HR}(\mathrm{p}=0.003)$. No other correlations were significant for maternal heart measures with maternal exercise measures.

We wanted to determine fetal echocardiographic changes within fetal activity state. When controlling for fetal activity state (FAS), 3 recordings did not have data available, thus leaving 47 recordings analyzed. Stratified by fetal activity state (active and quiet), there were no between-group differences in fetal heart measures (Table 3). 


\begin{tabular}{|c|c|c|c|c|c|c|}
\hline & \multicolumn{3}{|c|}{ ACTIVE activity state } & \multicolumn{3}{|c|}{ QUIET activity state } \\
\hline & $\begin{array}{l}\text { Control } \\
(\mathrm{n}=18)\end{array}$ & $\begin{array}{l}\text { Light Intensity } \\
\quad(n=15)\end{array}$ & p-value & $\begin{array}{l}\text { Control } \\
(\mathrm{n}=6)\end{array}$ & $\begin{array}{l}\text { Light Intensity } \\
(\mathrm{n}=8)\end{array}$ & p-value \\
\hline \multicolumn{7}{|l|}{ Fetal Heart Measures } \\
\hline Fetal HR (bpm) & $141.44(11.97)$ & $139.57(9.78)$ & 0.63 & $129.67(14.17)$ & $134.13(10.95)$ & 0.54 \\
\hline \multicolumn{7}{|c|}{ Right ventricular Heart Measures } \\
\hline RV Stroke Volume (L) & $1.00(0.15)$ & $1.00(0.16)$ & 0.86 & $1.14(0.24)$ & $1.14(0.14)$ & 0.98 \\
\hline $\begin{array}{l}\text { RV Stroke Index } \\
(\mathrm{mL} / \mathrm{kg})\end{array}$ & $0.38(0.06)$ & $0.37(0.07)$ & 0.76 & $0.40(0.06)$ & $0.40(0.06)$ & 0.85 \\
\hline $\begin{array}{l}\text { RV Cardiac Output } \\
(\mathrm{mL} / \mathrm{min})\end{array}$ & $141.17(15.91)$ & $139.59(19.43)$ & 0.81 & $142.15(19.80)$ & $153.16(21.03)$ & 0.37 \\
\hline $\begin{array}{l}\text { RV Cardiac Index } \\
(\mathrm{mL} / \mathrm{min} / \mathrm{kg})\end{array}$ & $53.57(8.63)$ & $51.84(8.97)$ & 0.60 & $49.96(6.45)$ & $53.929 .95)$ & 0.42 \\
\hline $\begin{array}{l}\text { RV Ejection Fraction } \\
(\%)\end{array}$ & $63.87(15.66)$ & $56.45(16.08)$ & 0.19 & $50.47(14.26)$ & $56.19(16.36)$ & 0.51 \\
\hline \multicolumn{7}{|c|}{ Left ventricular Heart Measures } \\
\hline LV Stroke Volume (L) & $0.88(0.22)$ & $0.87(0.23)$ & 0.84 & $1.08(0.41)$ & $0.90(0.18)$ & 0.39 \\
\hline $\begin{array}{l}\text { LV Stroke Index } \\
(\mathrm{mL} / \mathrm{kg})\end{array}$ & $0.34(0.08)$ & $0.32(0.08)$ & 0.65 & $0.37(0.10)$ & $0.33(0.04)$ & 0.47 \\
\hline $\begin{array}{l}\text { LV Cardiac Output } \\
(\mathrm{mL} / \mathrm{min})\end{array}$ & $125.88(26.84)$ & $120.72(32.11)$ & 0.63 & $134.11(43.10)$ & $119.79(16.36)$ & 0.51 \\
\hline $\begin{array}{l}\text { LV Cardiac Index } \\
(\mathrm{mL} / \mathrm{min} / \mathrm{kg})\end{array}$ & $47.57(10.24)$ & $44.96(11.65)$ & 0.52 & $46.14(10.59)$ & $43.88(4.33)$ & 0.67 \\
\hline $\begin{array}{l}\text { LV Ejection Fraction } \\
(\%)\end{array}$ & $65.12(14.79)$ & $67.13(14.39)$ & 0.70 & $61.76(24.53)$ & $59.53(18.01)$ & 0.86 \\
\hline $\begin{array}{l}\text { LV Fractional } \\
\text { Shortening }\end{array}$ & $44.55(9.40)$ & $41.90(10.53)$ & 0.46 & $39.72(16.59)$ & $37.99(15.58)$ & 0.85 \\
\hline \multicolumn{7}{|l|}{ Heart Valve Measures } \\
\hline $\begin{array}{l}\text { Pulmonary Valve Area } \\
\left(\mathrm{cm}^{2}\right)\end{array}$ & $79.41(16.99)$ & $77.70(17.56)$ & 0.78 & $87.50(23.58)$ & $90.25(15.35)$ & 0.82 \\
\hline $\begin{array}{l}\text { Pulmonary Valve Peak } \\
\text { Velocity }(\mathrm{cm} / \mathrm{s})\end{array}$ & $83.35(15.74)$ & $81.47(12.03)$ & 0.70 & $83.00(10.26)$ & 81.25 (11.09) & 0.77 \\
\hline $\begin{array}{l}\text { Pulmonary Valve Flow } \\
\text { Velocity }(\mathrm{cm} / \mathrm{s})\end{array}$ & $99.65(12.00)$ & $102.13(14.97)$ & 0.61 & $109.83(14.06)$ & $107.25(12.60)$ & 0.73 \\
\hline $\begin{array}{l}\text { Aortic Valve Area } \\
\left(\mathrm{cm}^{2}\right)\end{array}$ & $53.09(11.24)$ & $49.97(14.91)$ & 0.51 & $53.67(9.91)$ & $53.59(14.33)$ & 0.99 \\
\hline $\begin{array}{l}\text { Aortic Valve Peak } \\
\text { Velocity }(\mathrm{cm} / \mathrm{s})\end{array}$ & $101.24(17.73)$ & $98.40(11.02)$ & 0.59 & $105.17(17.58)$ & $96.25(7.87)$ & 0.29 \\
\hline $\begin{array}{l}\text { Aortic Valve Flow } \\
\text { Velocity }(\mathrm{cm} / \mathrm{s})\end{array}$ & $107.26(22.49)$ & $108.53(16.55)$ & 0.86 & $129.67(37.90)$ & $110.63(19.46)$ & 0.30 \\
\hline $\begin{array}{l}\text { Aortic } \\
\text { Valve/Pulmonary Valve }\end{array}$ & $0.82(0.09)$ & $0.81(0.12)$ & 0.78 & $0.80(0.12)$ & $0.77(0.11)$ & 0.72 \\
\hline \multicolumn{7}{|l|}{$\begin{array}{l}\text { Right : Left Ventricular } \\
\text { Associations }\end{array}$} \\
\hline RV CO: LV CO & $1.17(0.27)$ & $1.22(0.32)$ & 0.61 & $1.51(0.40)$ & $1.30(0.28)$ & 0.48 \\
\hline RV CI : LV CI & $1.17(0.27)$ & $1.22(0.32)$ & 0.61 & $1.15(0.40)$ & $1.23(0.19)$ & 0.70 \\
\hline $\begin{array}{l}\text { Combined CO } \\
(\mathrm{mL} / \mathrm{min})\end{array}$ & $267.05(33.80)$ & $260.31(41.89)$ & 0.63 & $276.26(50.79)$ & $272.95(25.70)$ & 0.90 \\
\hline $\begin{array}{l}\text { Combined CI } \\
(\mathrm{mL} / \mathrm{min} / \mathrm{kg})\end{array}$ & $101.14(15.63)$ & $96.80(17.00)$ & 0.48 & $96.11(7.60)$ & $97.80(12.75)$ & 0.78 \\
\hline
\end{tabular}


Controlling for FAS, significant correlations were found between maternal measures of fitness (peak VO2, 36 wk mHR) with fetal echocardiographic measures. In the quiet state, maternal fitness level (peak VO2) is inversely associated with pulmonary valve peak velocity (rho $=-0.559,0.047)$. Also in the quiet state, maternal resting HR at 36 weeks is correlated with fetal LV stroke index (rho=0.581, 0.047), LV cardiac index $(\mathrm{rho}=0.620,0.03)$, pulmonary valve peak velocity (rho $=0.692,0.01)$, pulmonary valve flow velocity $(0.606,0.02)$, and combined cardiac index $(\mathrm{rho}=0.640,0.03)$. In the active state, maternal fitness level was moderately associated with fetal LV stroke volume (rho $=0.458,0.01)$ and aortic valve area (rho=0.447, 0.01). In the active state, maternal resting $\mathrm{HR}$ at 36 weeks is negatively correlated with $\mathrm{LV}$ stroke index $(\mathrm{rho}=-0.379,0.04)$

We found many significant predictors for fetal heart function outcomes. For example, fetal RV cardiac output is predicted by FAS, as well as exercise volume; this significance is primarily driven by exercise duration (Table 4). Interestingly, gender predicts fetal RV ejection fraction (Table 4). Lastly, maternal exercise intensity predicts aortic valve flow velocity (Table 4). Thus, duration of maternal exercise is a predictor of right ventricular fetal heart function while intensity influences left ventricular blood flow velocity.

\begin{tabular}{|c|c|c|c|c|}
\hline & p-values & $\begin{array}{c}95 \% \text { CI } \\
\text { Lower Bound }\end{array}$ & $\begin{array}{c}95 \% \text { CI } \\
\text { Upper Bound }\end{array}$ & $\begin{array}{c}\text { Unstandardized Beta } \\
\text { Value }\end{array}$ \\
\hline RV Cardiac Output & \multicolumn{4}{|c|}{$p=0.02$ adjusted $R^{2}=0.27$} \\
\hline Fitness Level & 0.06 & -2.50 & 0.03 & -1.23 \\
\hline 36 wk Resting mSBP & 0.63 & -0.43 & 0.71 & 0.14 \\
\hline Exercise Volume & 0.01 & 0.01 & 0.07 & 0.04 \\
\hline Fetal Activity State & 0.02 & -23.57 & -2.15 & -12.86 \\
\hline Gravida & 0.12 & -1.21 & 9.96 & 4.38 \\
\hline RV Cardiac Output & \multicolumn{4}{|c|}{$p=0.03$ adjusted $R^{2}=0.25$} \\
\hline Fitness Level & 0.08 & -2.57 & 0.16 & -1.21 \\
\hline 36 wk Resting mHR & 0.95 & -0.34 & 0.32 & -0.01 \\
\hline Exercise Duration & 0.02 & 0.02 & 0.19 & 0.10 \\
\hline Fetal Activity State & $\mathbf{0 . 0 2}$ & -23.84 & -1.84 & -12.84 \\
\hline Gravida & 0.15 & -1.50 & 9.85 & 4.17 \\
\hline RV Ejection Fraction & \multicolumn{4}{|c|}{$p=0.04$ adjusted $R^{2}=0.25$} \\
\hline 36 wk Resting mHR & 0.16 & -0.09 & 0.50 & 0.21 \\
\hline 36 wk Resting mSBP & 0.07 & -0.03 & 0.93 & 0.45 \\
\hline Exercise Volume & 0.35 & -0.02 & 0.04 & 0.01 \\
\hline Fetal Activity State & 0.57 & -7.50 & 13.40 & 2.95 \\
\hline Gender & $\mathbf{0 . 0 3}$ & -20.45 & -1.42 & -10.93 \\
\hline RV Ejection Fraction & \multicolumn{4}{|c|}{$p=0.04$ adjusted $R^{2}=0.25$} \\
\hline 36 wk Resting mHR & 0.14 & -0.07 & -0.51 & 0.22 \\
\hline 36 wk Resting mSBP & 0.08 & -0.05 & 0.92 & 0.44 \\
\hline Exercise Duration & 0.51 & -0.05 & 0.11 & 0.03 \\
\hline Fetal Activity State & 0.56 & -7.43 & 13.59 & 3.08 \\
\hline Gender & 0.03 & -20.21 & -1.11 & -10.66 \\
\hline Aortic Valve Flow Velocity & \multicolumn{4}{|c|}{$p=0.02$ adjusted $R^{2}=0.19$} \\
\hline Prepregnancy BMI & 0.16 & -1.86 & 0.32 & -0.78 \\
\hline Exercise Intensity & $\mathbf{0 . 0 3}$ & -26.50 & -1.70 & -14.09 \\
\hline Fetal Activity State & 0.21 & -21.86 & 4.96 & -8.45 \\
\hline
\end{tabular}

Table 4: Regression analyses for Fetal Heart Function Measures

\section{Discussion}

We hypothesized that light intensity exercise during pregnancy would result in improved maternal resting heart measures and greater fetal cardiac function (i.e., increases in stroke volume and index, cardiac output and index, ejection fraction) but not fetal anatomical heart changes (i.e., pulmonary and aortic valve diameters) at 36 weeks of gestation compared to fetuses of non-exercising women. Our main finding shows that 1) maternal exercise intensity influences fetal left ventricular heart flow while 2) exercise duration influences fetal right ventricular outcomes. As hypothesized, there were no differences in fetal cardiac anatomical 
measures. Lastly, light intensity exercise and duration of exercise are important for maternal cardiovascular benefits during pregnancy.

In support of our hypothesis, we found light intensity exercise influences fetal left ventricular outflow. These findings align with earlier research that shows a dose-response relationship between maternal exercise intensity and offspring cardiac adaptations [9, 23, 46, 47]. Though the differences compared to controls in fetal flow velocities were not as large in this analysis, the findings are similar to the significant left ventricular flow velocity observed in fetuses exposed to moderate intensity aerobic exercise [1]. Importantly, left ventricular outflow measures are thought to contribute to the atherosclerotic environment [48] and are a better predictor of adverse cardiovascular outcomes than left ventricular ejection fraction [38]. Although the changes are smaller; they demonstrate potentially decreased risk of adverse cardiovascular outcomes for the fetus due to maternal light intensity exercise. The smaller differences from maternal light intensity may be explained, in part, by higher intensities stimulating changes in both sympathetic and parasympathetic cardiac activity while low intensities lead to cardiac changes predominantly due to withdrawal of parasympathetic activity. Since the fetal cardiac ANS is developing, the difference in outcomes from exercise intensity may indicate different physiological responses needed to activate and mature these two autonomic nervous system branches [49, 50]. Interestingly, exercise during pregnancy increases serum and placenta superoxide dismutase (SOD), which breaks down free radicals and can regulate genes, DNA methylation, and cell functions [51]. Since exercise of any intensity or duration is associated with increased SOD in the aorta [51], this placental response may explain some of the changes associated with light intensity maternal exercise on fetal left ventricular function.

Exercise duration influenced fetal right ventricular outcomes. Comparable to differences in fetal heart measures due to supervised, moderate intensity maternal exercise[1], light intensity exercise at the recommended duration of 150 minutes each week influenced fetal right ventricular heart outcomes. Potentially due to the lower energy expenditure associated with lower intensity, a longer duration of lower intensity may elicit a similar response as the 150 minutes of moderate intensity exercise[21]. When total energy is held constant, longer sessions of moderate intensity exercise has similar cardioprotective benefits as shorter bouts of vigorous intensity exercise.[52] Similarly when energy expenditure is constant, light intensity exercise of longer duration improved insulin and plasma lipids more than shorter sessions of moderate-to-vigorous exercise in sedentary non-gravid adults.[53] Others have shown exercise duration associated with bioavailability of endothelial nitric oxide and vascular collateralization [54-56]. This effect may influence the placenta, which would then impact the fetal right heart as it is the primary heart pump in utero. Therefore, this outcome calls for further study.

Our study additionally shows that light intensity exercise and duration of exercise elicits changes coincident with improved maternal cardiovascular function (e.g., maternal blood pressure). An echocardiographic study comparing light-moderate exercise to no exercise found no significant differences but did reveal trends of lower resting BP and HR with slightly increased stroke volume and cardiac output at 34 weeks [57]. The current findings are similar to a study of overweight and normal weight women that demonstrated improved blood pressure and HRV due to light intensity walking throughout pregnancy [58]. Light intensity physical activity is associated with improved maternal diastolic blood pressure[20], submaximal aerobic capacity [59], decreased blood pressure and catecholamines [60], increased antioxidants (superoxide dismutase, transferrin) [18], and decreased cardiometabolic risk [61], such as improved blood lipids, glucose and insulin levels, Cpeptide levels, C-reactive protein (CRP), decreased intima-media thickness, restored diastolic function and cardiac synchrony, as well as improved waist circumference and body composition without weight loss in adults [62-67]. Additionally, in rats, light intensity exercise has been shown to increase action potential amplitude, a determinant of actional potential velocity across myocardium more than moderate intensity and controls [68]. It is possible that stretching and light intensity exercise while pregnant enhance maternal cardiac autonomic control, which may reduce the risks for certain conditions, such as preeclampsia/gestational hypertension, gestational diabetes [14, 17, 69]. Light intensity exercise in resistant hypertensive adults has been shown to have BP benefits after one session and benefits persist longer after the session than moderate intensity exercise [67]. Similar to our findings, light intensity stretching during pregnancy was not associated with adverse pregnancy outcomes; further, studies have found a dose-response relationship for the duration of the light intensity exercise and decreased adverse outcomes [69] and CRP [20,70]. Meta-analyses have noted exercise intensity, duration, and volume are associated with reduced risk of pregnancy complications [71]. In non-pregnant populations, duration of light intensity exercise is positively related to cardiometabolic improvements [72-74]. Additionally, the potentially increased adherence to and preference of light intensity exercise during pregnancy, especially in specific populations (e.g., Hispanic, Black, obese, hypertensive, multifetal gestation), makes this exercise a potential therapeutic alternative for improving maternal outcomes and warrants further investigation $[11,12]$.

The strengths of this study include the focus on women performing light intensity exercise during pregnancy. To the best of our knowledge, this was the first analysis that examined the effects of supervised light intensity exercise during pregnancy on specific fetal cardiac function measures. We controlled for time of day of the fetal recording and fetal activity state; additionally, all women were instructed to eat a snack 1 to 1.5 hours prior to the recording. There were also several limitations and methodological biases to this study. First, our light intensity exercise was an exercise dose less than the recommendations for pregnant women; thus, due to the lower MET and energy expenditure associated with lower intensity, the light intensity exercise protocol most likely should have been of longer duration[21], warranting further study. Secondly, although our sample size for per protocol analysis was small, we had sufficient power to test our hypothesis. Similarly, there was sufficient data in the fetal active state to test the hypothesis. Next, because we did not account for potential differences in maternal nutrition, it is possible that there are dietary differences, which may have contributed to differences in fetal heart development. For example, docosahexaenoic acid (DHA), which is an essential fatty acid for fetal nervous system development, could account for some differences. Lastly, there is a lack of longitudinal measures to find if changes are measurable after birth, and further research is needed after birth to determine if dose response changes in the offspring heart development persist.

\section{Conclusions}

The current study has interesting and new findings for this area of research. We found that maternal light intensity exercise and its duration improve fetal heart outcomes. Furthermore, light intensity exercise also has beneficial changes on maternal heart measures. Therefore, light intensity exercise benefits both mother and fetus and may be more tolerated in specific populations.

\section{Conflict of Interest}

The authors declare that the research was conducted in the absence of any commercial or financial relationships that could be construed as a potential conflict of interest.

\section{Author Contributions}

EN and CI provided oversight for all participants in the study. DS completed all fetal echocardiographic recordings. LS and RDS oversaw 
the echocardiographic interpretation of the recordings. CJ and SM completed the statistical analysis. BD and SM drafted the manuscript. SY and DK provided oversight on data interpretation and manuscript revisions. LM is the lead author who designed and oversaw all aspects of the study, data collection, data interpretation, and writing. All authors contributed to this work.

\section{Funding}

Supported, in part, by the American Heart Association (AHA grant \#15GRNT24470029) and by East Carolina University internal funds.

\section{Acknowledgments}

We thank the women who participated in this study and who gave their time and effort.

\section{References}

1. May, L.E., et al., Influence of maternal aerobic exercise during pregnancy on fetal cardiac function and outflow. Am J Obstet Gynecol MFM, 2020. 2(2): p. 100095.

2. Laredo-Aguilera, J.A., et al., Physical Activity Programs during Pregnancy Are Effective for the Control of Gestational Diabetes Mellitus. Int J Environ Res Public Health, 2020. 17(17).

3. Zheng, J., L.Y. Zhou, and X.H. Xiao, Maternal exercise and its beneficial effects on glucose metabolism in offspring. Chin Med J (Engl), 2020. 133(7): p. 863-867.

4. Yang, S.Y., et al., Effects of Exercise on Sleep Quality in Pregnant Women: A Systematic Review and Meta-analysis of Randomized Controlled Trials. Asian Nurs Res (Korean Soc Nurs Sci), 2020. 14(1): p. 1-10.

5. Davenport, M.H., et al., Impact of prenatal exercise on maternal harms, labour and delivery outcomes: a systematic review and meta-analysis. Br J Sports Med, 2019. 53(2): p. 99-107.

6. Skow, R.J., et al., Effects of prenatal exercise on fetal heart rate, umbilical and uterine blood flow: a systematic review and metaanalysis. Br J Sports Med, 2019. 53(2): p. 124-133.

7. Wiebe, H.W., et al., The effect of supervised prenatal exercise on fetal growth: a meta-analysis. Obstet Gynecol, 2015. 125(5): p. 1185-1194.

8. Hesketh, K.R. and K.R. Evenson, Prevalence of U.S. Pregnant Women Meeting 2015 ACOG Physical Activity Guidelines. Am J Prev Med, 2016. 51(3): p. e87-89.

9. May, L.E., et al., Regular maternal exercise dose and fetal heart outcome. Med Sci Sports Exerc, 2012. 44(7): p. 1252-1258.

10. Yeo, S., A risk reduction model for late-onset preeclampsia: a theory for using low-intensity exercises to enhance cardiac homeostasis in nursing research and practice. ANS Adv Nurs Sci, 2011. 34(1): p. 78-88.

11. Catov, J.M., et al., Patterns of leisure-time physical activity across pregnancy and adverse pregnancy outcomes. Int J Behav Nutr Phys Act, 2018. 15(1): p. 68.

12. Kim, Y. and E. Chung, Descriptive Epidemiology of Objectively Measured Walking Among US Pregnant Women: National Health and Nutrition Examination Survey, 2005-2006. Prev Chronic Dis, 2015. 12: p. E217.

13. Yeo, S., Adherence to walking or stretching, and risk of preeclampsia in sedentary pregnant women. Res Nurs Health, 2009. 32(4): p. 379-390.

14. Yeo, S., Prenatal stretching exercise and autonomic responses: preliminary data and a model for reducing preeclampsia. J Nurs Scholarsh, 2010. 42(2): p. 113-121.

15. Yeo, S., et al., A comparison of walking versus stretching exercises to reduce the incidence of preeclampsia: a randomized clinical trial. Hypertens Pregnancy, 2008. 27(2): p. 113-130.

16. Logan, J.G. and S. Yeo, Effects of Stretching Exercise on Heart Rate Variability During Pregnancy. J Cardiovasc Nurs, 2017. 32(2): p. 107-111.

17. Satyapriya, M., et al., Effect of integrated yoga on stress and heart rate variability in pregnant women. Int J Gynaecol Obstet, 2009. 104(3): p. 218-222.

18. Sankaralingam, S., et al., Effect of exercise on vascular superoxide dismutase expression in high-risk pregnancy. Am J Perinatol, 2011. 28(10): p. 803-810.

19. Poyatos-Leon, R., et al., Effects of exercise during pregnancy on mode of delivery: a meta-analysis. Acta Obstet Gynecol Scand, 2015. 94(10): p. 1039-1047.

20. Loprinzi, P.D., et al., Association of physical activity and sedentary behavior with biological markers among U.S. pregnant women. J Womens Health (Larchmt), 2013. 22(11): p. 953-958.

21. Ruchat, S.M., et al., Effect of exercise intensity and duration on capillary glucose responses in pregnant women at low and high risk for gestational diabetes. Diabetes Metab Res Rev, 2012. 28(8): p. 669-678.

22. Bolanthakodi, C., et al., Prenatal Yoga: Effects on Alleviation of Labor Pain and Birth Outcomes. J Altern Complement Med, 2018. 24(12): p. 1181-1188.

23. May, L., Suminski, RS., Amount of Physical Activity in Pregnancy and Infant heart Outcomes. Journal of Neonatal Biology, 2014. in press.

24. Gynecologists, A.C.o.O.a., Physical Activity and Exercise During Pregnancy and the Postpartum Period. ACOG Committee Opinion, 2015. 650: p. 8.

25. Mottola, M.F., et al., VO2peak prediction and exercise prescription for pregnant women. Med Sci Sports Exerc, 2006. 38(8): p. 1389-1395.

26. Swain, D.P. and American College of Sports Medicine., ACSM's resource manual for Guidelines for exercise testing and prescription. 7th ed. 2014, Philadelphia: Wolters Kluwer Health/Lippincott Williams \& Wilkins. xv, 862 p.

27. Kriska, A.M., et al., Development of questionnaire to examine relationship of physical activity and diabetes in Pima Indians. Diabetes Care, 1990. 13(4): p. 401-411.

28. Cramp, A.G. and S.R. Bray, A prospective examination of exercise and barrier self-efficacy to engage in leisure-time physical activity during pregnancy. Ann Behav Med, 2009. 37(3): p. 325-334.

29. Cramp, A.G. and S.R. Bray, Pre- and postnatal women's leisure time physical activity patterns: a multilevel longitudinal analysis. Res Q Exerc Sport, 2009. 80(3): p. 403-411.

30. Schulz, L.O., et al., Energy intake and physical activity in Pima Indians: comparison with energy expenditure measured by doubly-labeled water. Obes Res, 1994. 2(6): p. 541-548.

31. Bauer, P.W., C.L. Broman, and J.M. Pivarnik, Exercise and pregnancy knowledge among healthcare providers. J Womens Health (Larchmt), 2010. 19(2): p. 335-341.

32. Davies, G.A., et al., Joint SOGC/CSEP clinical practice guideline: exercise in pregnancy and the postpartum period. Can J Appl Physiol, 2003. 28(3): p. 330-341.

33. Ainsworth, B.E., et al., 2011 Compendium of Physical Activities: a second update of codes and MET values. Med Sci Sports Exerc, 2011. 43(8): p. 1575-1581.

34. Molina, F.S., et al., Heart stroke volume and cardiac output by four-dimensional ultrasound in normal fetuses. Ultrasound Obstet Gynecol, 2008. 32(2): p. 181-187. 
35. Simioni, C., et al., Fetal cardiac function assessed by spatiotemporal image correlation. Arch Gynecol Obstet, 2011. 284(1): p. 253-260.

36. Vintzileos, A.M., et al., The ultrasound femur length as a predictor of fetal length. Obstet Gynecol, 1984. 64(6): p. 779782.

37. Miller, A. and J. Mandeville, Predicting and measuring fluid responsiveness with echocardiography. Echo Res Pract, 2016. 3(2): p. G1-G12.

38. Tan, C., et al., Left ventricular outflow tract velocity time integral outperforms ejection fraction and Doppler-derived cardiac output for predicting outcomes in a select advanced heart failure cohort. Cardiovasc Ultrasound, 2017. 15(1): p. 18.

39. Mosteller, R.D., Simplified calculation of body-surface area. $\mathrm{N}$ Engl J Med, 1987. 317(17): p. 1098.

40. Nijhuis, I.J., et al., Fetal heart rate in relation to its variation in normal and growth retarded fetuses. Eur J Obstet Gynecol Reprod Biol, 2000. 89(1): p. 27-33.

41. Pillai, M. and D. James, The development of fetal heart rate patterns during normal pregnancy. Obstet Gynecol, 1990. 76(5 Pt 1): p. 812-816.

42. Ten Hof, J., et al., Longitudinal study of fetal body movements: nomograms, intrafetal consistency, and relationship with episodes of heart rate patterns a and B. Pediatr Res, 2002. 52(4): p. 568-575.

43. Prevention, C.f.D.C.a. About Adult BMI | Healthy Weight, Nutrition, and Physical Activity | CDC. 2020 [cited 2020 November 19, 2020]; See how body mass index is used and how to interpret adult results.].

44. Moyer, C., et al., Influence of exercise mode on pregnancy outcomes: ENHANCED by Mom project. BMC Pregnancy Childbirth, 2015. 15: p. 133.

45. Murphy, S., et al., Influence of Exercise Type on Maternal Blood Pressure Adaptation Throughout Pregnancy. AJOG Global Reports, 2021. in print.

46. May, L., Glaros, AG, Yeh, H-W, Clapp, JF, Gustafson, KM, Aerobic Exercise during Pregnancy Influences Fetal Cardiac Autonomic Control of Heart Rate and Heart Rate Variability. Early Hum Dev, 2010. 86(4): p. 17.

47. May, L.E., et al., Aerobic exercise during pregnancy influences infant heart rate variability at one month of age. Early Hum Dev, 2014. 90(1): p. 33-38.

48. Rodríguez, I. and M. González, Physiological mechanisms of vascular response induced by shear stress and effect of exercise in systemic and placental circulation. Front Pharmacol, 2014. 5: p. 209.

49. Rowell, L.B. and D.S. O'Leary, Reflex control of the circulation during exercise: chemoreflexes and mechanoreflexes. J Appl Physiol (1985), 1990. 69(2): p. 407-418.

50. Schneider, U., et al., Developmental milestones of the autonomic nervous system revealed via longitudinal monitoring of fetal heart rate variability. PLoS One, 2018. 13(7): p. e0200799.

51. Kusuyama, J., et al., Placental superoxide dismutase 3 mediates benefits of maternal exercise on offspring health. Cell Metab, 2021. 33(5): p. 939-956.e8.

52. Swain, D.P. and B.A. Franklin, Comparison of cardioprotective benefits of vigorous versus moderate intensity aerobic exercise. Am J Cardiol, 2006. 97(1): p. 141-147.

53. Duvivier, B.M., et al., Minimal intensity physical activity (standing and walking) of longer duration improves insulin action and plasma lipids more than shorter periods of moderate to vigorous exercise (cycling) in sedentary subjects when energy expenditure is comparable. PLoS One, 2013. 8(2): p. e55542.

54. Lee, S., Y. Park, and C. Zhang, Exercise Training Prevents Coronary Endothelial Dysfunction in Type 2 Diabetic Mice. Am J Biomed Sci, 2011. 3(4): p. 241-252.

55. Heaps, C.L. and J.L. Parker, Effects of exercise training on coronary collateralization and control of collateral resistance. J Appl Physiol (1985), 2011. 111(2): p. 587-98.

56. Hambrecht, R., et al., Regular physical activity improves endothelial function in patients with coronary artery disease by increasing phosphorylation of endothelial nitric oxide synthase. Circulation, 2003. 107(25): p. 3152-3158.

57. Perales, M., et al., Maternal Cardiac Adaptations to a Physical Exercise Program during Pregnancy. Med Sci Sports Exerc, 2016. 48(5): p. 896-906.

58. Stutzman, S.S., et al., The effects of exercise conditioning in normal and overweight pregnant women on blood pressure and heart rate variability. Biol Res Nurs, 2010. 12(2): p. 137-148.

59. Ruchat, S.M., et al., Walking program of low or vigorous intensity during pregnancy confers an aerobic benefit. Int $\mathbf{J}$ Sports Med, 2012. 33(8): p. 661-666.

60. Dempsey, P.C., et al., Interrupting prolonged sitting with brief bouts of light walking or simple resistance activities reduces resting blood pressure and plasma noradrenaline in type 2 diabetes. J Hypertens, 2016. 34(12): p. 2376-2382.

61. Whitaker, K.M., et al., Associations of AccelerometerMeasured Sedentary Time and Physical Activity With Prospectively Assessed Cardiometabolic Risk Factors: The CARDIA Study. J Am Heart Assoc, 2019. 8(1): p. e010212.

62. Sjöros, T., et al., Both sedentary time and physical activity are associated with cardiometabolic health in overweight adults in a 1 month accelerometer measurement. Sci Rep, 2020. 10(1): p. 20578.

63. Ryan, D., et al., Segregating the Distinct Effects of Sedentary Behavior and Physical Activity on Older Adults' Cardiovascular Structure and Function: Part 1-Linear Regression Analysis Approach. J Phys Act Health, 2018. 15(7): p. 499-509.

64. Schuster, I., et al., Diastolic dysfunction and intraventricular dyssynchrony are restored by low intensity exercise training in obese men. Obesity (Silver Spring), 2012. 20(1): p. 134-140.

65. Del Pozo-Cruz, J., et al., Replacing Sedentary Time: Metaanalysis of Objective-Assessment Studies. Am J Prev Med, 2018. 55(3): p. 395-402.

66. Dempsey, P.C., et al., Benefits for Type 2 Diabetes of Interrupting Prolonged Sitting With Brief Bouts of Light Walking or Simple Resistance Activities. Diabetes Care, 2016. 39(6): p. 964-972.

67. Santos, L.P., et al., Effects of aerobic exercise intensity on ambulatory blood pressure and vascular responses in resistant hypertension: a crossover trial. J Hypertens, 2016. 34(7): p. 1317-1324.

68. Batacan, R.B., et al., Light-intensity and high-intensity interval training improve cardiometabolic health in rats. Appl Physiol Nutr Metab, 2016. 41(9): p. 945-952.

69. Yeo, S. and J.H. Kang, Low-Intensity Exercise and Pregnancy Outcomes: An Examination in the Nurses' Health Study II. Womens Health Rep (New Rochelle), 2021. 2(1): p. 389-395.

70. Hawkins, M., P. Pekow, and L. Chasan-Taber, Physical activity, sedentary behavior, and C-reactive protein in pregnancy. Med Sci Sports Exerc, 2014. 46(2): p. 284-292.

71. Cai, C., et al., Prenatal Exercise and Cardiorespiratory Health and Fitness: A Meta-analysis. Med Sci Sports Exerc, 2020. 52(7): p. 1538-1548. 
72. Jefferis, B.J., et al., Does duration of physical activity bouts matter for adiposity and metabolic syndrome? A cross-sectional study of older British men. Int J Behav Nutr Phys Act, 2016. 13: p. 36.

73. Loprinzi, P.D. and M. Kohli, Effect of physical activity and sedentary behavior on serum prostate-specific antigen concentrations: results from the National Health and Nutrition Examination Survey (NHANES), 2003-2006. Mayo Clin Proc, 2013. 88(1): p. 11-21.

74. Robson, J. and I. Janssen, Intensity of bouted and sporadic physical activity and the metabolic syndrome in adults. PeerJ, 2015. 3: p. e1437.
Ready to submit your research? Choose Auctores and benefit from:

$>$ fast, convenient online submission

$>$ rigorous peer review by experienced research in your field

$>$ rapid publication on acceptance

$>$ authors retain copyrights

$>$ unique DOI for all articles

$>$ immediate, unrestricted online access

At Auctores, research is always in progress.

Learn more https://auctoresonline.org/journals/clinical-cardiology-andcardiovascular-interventions 